\title{
ESTUDO COMPARATIVO DA QUALIDADE DE VIDA SEXUAL EM INDIVÍDUOS COM LESÃO MEDULAR: PARATLETAS E NÃO PARATLETAS
}

\author{
Lissa Oliveira Abreu,' Geovanna Romana Matos Amaral Ferreira, ${ }^{2}$ Wendel de Lima Albuquerque, ${ }^{3}$ \\ Iranete Corpes Oliveira França, ${ }^{4}$ Caren Helouise da Costa Priante, ${ }^{5}$ Susanne Cristine Brito e Silva, ${ }^{6}$ \\ Claudia Jeane Claudino de Pontes Miranda ${ }^{7}$
}

\author{
COMPARATIVE STUDY OF THE QUALITY OF SEXUAL LIFE IN INDIVIDUALS WITH \\ SPINAL INJURY: ATHLETES AND NON-PARAPLEGIC ATHLETES
}

\section{ESTUDIO COMPARATIVO DE LA CALIDAD DE VIDA SEXUAL EN PERSONAS CON LESIÓN ESPINAL: ATLETAS Y ATLETAS NO PARAAPLÉGICOS}

\begin{abstract}
Resumo: A lesão medular (LM) é responsável por ocasionar danos neurológicos que interferem na autonomia de um indivíduo, ela caracteriza-se por alterações motoras, sensitivas e do sistema nervoso autônomo que podem variar de acordo com localização da lesão. Dentre as consequências dessas alterações da LM estão as disfunções sexuais. Dispor de uma vida sexual ativa e saudável contribui para uma melhor qualidade de vida de um indivíduo. A função sexual é reconhecida pela Organização Mundial da Saude como um sinal de saúde, sendo assim, as disfunções sexuais são consideradas um problema de saúde pública. A prática esportiva é um recurso importante para a melhora da funcionalidade, autoestima, autoaceitação, prevenir o desenvolvimento de limitações funcionais secundárias, além da melhora da qualidade de vida sexual. O objetivo deste estudo é comparar a qualidade de vida sexual de paratletas e não paratletas. O tipo de pesquisa é descritivo, utilizando métodos quantitativos, na mensuração das frequências absolutas e relativas. Os dados foram colhidos através de um questionário de sexualidade humana para indivíduos com lesão medular (QSH-LM modificado). Nos resultados, adotou-se para realização do teste um nível de significância de $p$-valor $<0.05 ; 92,3 \%$ eram muito satisfeitos antes da $L M(p=0,3352)$. Depois da lesão, a satisfação sexual, que está ligada à qualidade de vida sexual, $43 \%$ dos não paratletas relataram estar moderadamente satisfeitos $(p=0,04 \mathrm{I})$ enquanto $50 \%$ dos atletas com LM assinalaram o mesmo quesito $(p=0,041)$. Somente $7,7 \%$ relataram estar muito satisfeitos depois da lesão medular $(p=0,6286)$.
\end{abstract}

Palavras-chave: lesão medular; qualidade de vida; sexualidade; atletas; não atletas.

Abstract: Spinal cord injury $(\mathrm{SCl})$ is responsible for causing neurological damage that interferes with an individual's autonomy, it is characterized by motor, sensory and in the autonomic nervous system changes that can differ according to the injury's location. Among the consequences of these changes in $\mathrm{SCl}$, are the sexual dysfunctions. Having an active and healthy sex life contributes to a better quality of life for an individual. Sexual function is recognized by the World Health Organization as a sign of health, consequently, sexual dysfunctions are considered a public health problem.

\footnotetext{
Graduada em Fisioterapia pelo Centro de Ciências Biológicas e da Saúde da Universidade da Amazônia, Belém, Brasil. E-mail: Lissa.abreu22@gmail.com

2 Graduada em Fisioterapia pelo Centro de Ciências Biológicas e da Saúde da Universidade da Amazônia, Belém, Brasil. E-mail: Ferreirageovanna044@gmail.com

${ }^{3}$ Graduado em Fisioterapia pelo Centro de Ciências Biológicas e da Saúde da Universidade da Amazônia, Belém, Brasil. E-mail:Wendellima547@gmail.com

${ }^{4}$ Graduada em Fisioterapia pela Universidade do Estado do Pará e mestre em Desenvolvimento e Meio Urbano pela Universidade da Amazônia. Docente do Centro de Ciências Biológicas e da Saúde da Universidade da Amazônia, Belém, Brasil. E-mail: iranetecorpes@gmail.com

${ }^{5}$ Graduada em Fisioterapia pela Universidade da Amazônia, mestre em Ciências da Saúde pela Universidade Federal de são Paulo. Pesquisadora Autonôma. E-mail: caren_priante@hotmail.com

${ }^{6}$ Graduada em Fisioterapia pela Universidade do Estado do Pará e mestre em Teoria da Pesquisa do Comportamento pela Universidade Federal do Pará. Docente do Centro de Ciências Biológicas e da Saúde da Universidade da Amazônia, Belém, Brasil. E-mail: Susanne.fisiol23@gmail.com

7 Graduada em Fisioterapia pela Universidade Federal da Paraíba, mestre e doutora em Ciências Médicas pela Universidade de São Paulo. Docente do Centro de Ciências Biológicas e da Saúde, Universidade da Amazônia, Belém, Brasil. E-mail: cjpontes@terra.com.br
} 
(1) Revista Brasileira de

Sexualidade Humana

DOI: https://doi.org/10.35919/rbsh.v32il.962

Sports practice is an important resource for improving functionality, self-esteem, self-acceptance, preventing the development of secondary functional limitations, in addition to improving the quality of sexual life. The aim of this study is to compare the sexual quality of life of athletes and non-athletes who are paraplegic. The type of research is descriptive, using quantitative methods, in the measurement of absolute and relative frequencies. Data were collected using a human sexuality questionnaire for individuals with spinal cord injury (modified QSH-LM). In the results, a significance level of $\mathrm{p}$-value $<0.05$ was adopted for the test; $92.3 \%$ were very satisfied before $\mathrm{SCl}(\mathrm{p}=0.3352)$. After the injury, the sexual satisfaction, which is linked to the quality of sexual life, $43 \%$ of non-athlete paraplegics reported moderately satisfied $(p=0.04 \mathrm{I})$ while $50 \%$ of athletes with $\mathrm{SCl}$ indicated the same issue $(\mathrm{p}=0.04 \mathrm{I})$. Only $7.7 \%$ reported being very satisfied after spinal cord injury $(p=0.6286)$.

Keywords: spinal cord injury; quality of life; sexuality; athletes; not athletes.

Resumen: La lesión de la médula espinal (LME) es responsable de causar daño neurológico que interfiere con la autonomía de un individuo, se caracteriza por cambios en el sistema nervioso motor, sensorial y autónomo que pueden variar según la ubicación de la lesión. Entre las consecuencias de estos cambios en la LME se encuentran las disfunciones sexuales. Tener una vida sexual activa y saludable contribuye a una mejor calidad de vida para el individuo. La función sexual es reconocida por la OMS como un signo de salud, por lo que la disfunción sexual se considera un problema de salud pública. La práctica deportiva es un recurso importante para mejorar la funcionalidad, la autoestima, la autoaceptación, previniendo el desarrollo de limitaciones funcionales secundarias, además de mejorar la calidad de vida sexual. El objetivo de este estudio es comparar la calidad de vida sexual de deportistas y no deportistas parapléjicos. El tipo de investigación es descriptiva, utilizando métodos cuantitativos, midiendo frecuencias absolutas y relativas. Los datos se recopilaron mediante un cuestionario de sexualidad humana para personas con lesión de la médula espinal (QSH-LM modificado). En los resultados, se adoptó un nivel de significancia de p-value < 0.05 para la prueba; El 92,3\% se mostró muy satisfecho antes de la LME ( $p=0,3352)$. Después de la lesión, la satisfacción sexual, que está vinculada a la calidad de vida sexual, el $43 \%$ de los parapléjicos no deportistas reportaron satisfacción moderada $(p=0,04 \mathrm{I})$ mientras que el $50 \%$ de los deportistas con LME indicaron lo mismo ( $p=0,041)$. Solo el 7,7\% informó estar muy satisfecho después de la lesión de la médula espinal $(p=0,6286)$.

Keywords: lesión de la médula espinal; calidad de vida; sexualidad; atletas; no deportistas.

\section{Introdução}

A Lesão Medular (LM) é um problema de saúde pública, pois há um predomínio crescente na população e necessita de um atendimento individual, que inclui prevenção, proteção e recuperação da saúde. Além disso, a incapacidade e morbidade decorrentes do trauma interferem na qualidade de vida dos portadores da lesão medular e de seus familiares, visto que, dependendo da complexidade da lesão, o indivíduo fica dependente, os medicamentos aumentam o custo do Sistema Único de Saúde (SUS) e contribui para a redução da população economicamente ativa (JAMES et al., 2019).

No Brasil, ocorrem 40 novos casos de trauma raquimedular por milhão de habitantes a cada ano, ou aproximadamente 8 mil novos casos a cada ano. Os homens são mais suscetíveis a acidentes e traumas que podem desencadear a LM, pois são mais vulneráveis a atividades prejudiciais. Portanto, $80 \%$ dos casos são homens entre 15 e 35 anos (MIRANDA et al., 2016).

Diante disso, a lesão é caracterizada por alterações no sistema nervoso motor, sensorial e autônomo, que podem variar dependendo da localização da lesão. Além disso, essa lesão também pode desencadear outras alterações, como depressão, baixa autoestima, dificuldades de autoaceitação e disfunção sexual (CARDOSO et al., 2018).

Os indivíduos com LM muitas vezes passam por mudanças sexuais, e sua relação com o corpo e o ambiente externo pode interferir na imaginação, vontade e comportamento fisiológico do indivíduo. Atualmente, a orientação sobre transtornos sexuais, a prescrição de medicamentos para melhorar o desejo e a excitação e o uso de dispositivos artificiais para aumentar a lubrificação e a ereção são recursos e estratégias de tratamento para melhorar a vida sexual dessas pessoas, pois a atividade sexual é um fator de extrema importância para que alcancem a qualidade de vida ideal (KRASSIOUKOV; ELLIOTT, 20I7).

O exercício físico é um recurso importante para melhorar a função, a autoestima, a autoaceitação, evitando o desenvolvimento de limitações funcionais secundárias, incentivando a participação e a adaptação social, pois possibilitam às pessoas a troca de conhecimentos $\mathrm{e}$ experiências de vida com pessoas com deficiência física (FIDLER; SCHMIDT; VAUHNIK, 2017). 
A lesão causará danos ao indivíduo de acordo com a localização da lesão na coluna, e classifica-se em perda total ou parcial da atividade sensorial e/ou motora dos membros inferiores como paraparesia/paraplegia e, quando acomete os membros superiores e inferiores, chama-se tetraparesia/ tetraplegia. A avaliação de distúrbios motores e sensoriais é determinada de acordo com normas Internacionais para Classificação Neurológica de LM. Para tanto, os profissionais utilizam uma escala com escores padronizados de função motora, que também avalia a função sensorial, a American Spinal Injury Association (ASIA), para localizar o grau da lesão e sua integridade. A partir disso, observa-se as mudanças que podem ser acionadas (WALTER; KRASSIOUKOV, 2019).

O trabalho atual é de grande relevância, pois os estudos pesquisados para agregá-lo destacam a importância do exercício físico, visto que contribui para bem-estar geral, consequentemente, melhora a qualidade de vida sexual. No entanto, também é necessário correlacionar o grau e o tempo da lesão e o tempo com a qualidade de vida sexual, assim como a satisfação sexual dos praticantes e não praticantes de esportes deve ser comparada. Sendo assim, o objetivo do presente artigo é comparar a qualidade de vida sexual de paratletas e não paratletas, abordando se existe $\circ$ aconselhamento por parte dos profissionais de saúde relacionados a qualidade de vida sexual para as pessoas com LM e seus parceiros.

\section{Métodos}

Trata-se de um estudo transversal de carácter descritivo, utilizando métodos quantitativos. Na mensuração das frequências absolutas e relativas, a pesquisa quantitativa utilizada nesta pesquisa visa processar estatisticamente os dados para identificar tendências, aderências e correlações entre as variáveis da pesquisa.

Foram incluídos indivíduos maiores de 18 anos com lesão medular completa e incompleta abaixo de TI, que tinham cognição preservada e sofreram lesão predeterminada por um ano ou mais. Os critérios de exclusão foram quaisquer indícios que possam causar desvios nos resultados do projeto, como doenças mentais, pacientes diabéticos, fumantes ou traumas cerebrais.

Os dados foram coletados através de um questionário de sexualidade humana para indivíduos com lesão medular (QSH-LM modificado). O questionário é dividido em três partes, sendo a primeira composta por informações constantes dos dados pessoais dos participantes. A segunda parte contém informações sobre a qualidade da vida sexual antes da $L M$, e a terceira parte são informações sobre a vida sexual após a lesão. Composto por 60 perguntas fechadas e de múltiplas escolhas.

O questionário foi aplicado pelos pesquisadores de forma presencial ou pelo questionário on-line. Inicialmente os participantes assinaram o Termo de
Consentimento Livre e Esclarecido (TCLE). Seguido do instrumento de pesquisa, que é autoaplicável, garantindo, dessa forma, o anonimato. Os locais em que as coletas de dados foram realizadas são, Unidade de Referência Especializada Demétrio Medrado e All Star Rodas, ambos localizados na cidade de Belém, no Pará.

A realização da pesquisa foi de acordo com o código de Nuremberg, considerando os aspectos éticos em pesquisa com seres humanos, e em concordância com a resolução 466//2 do Conselho Nacional de Saúde. A pesquisa foi aprovada pelo Comitê de Ética em Pesquisa do Instituto Campinense de Ensino Superior-Universidade da Amazônia (CEP ICES-UNAMA) com o CAAE de número 32784620.1.0000.5 173. Sob número do parecer: 4.218.307.

Neste estudo foi utilizado o teste da razão de verossimilhança do Qui-quadrado para amostras independentes. Trata-se de um teste de hipótese que usa conceitos estatísticos para rejeitar ou não uma hipótese nula $\left(\mathbf{H}_{0}=\mathrm{As}\right.$ frequências observadas ocorrem na mesma proporção para os diferentes grupos). É um teste estatístico para $n$ amostras cujas proporções das diversas modalidades estão dispostas em tabelas de frequência, sendo os valores esperados deduzidos matematicamente, procurando-se determinar se as proporções observadas nas diferentes categorias ocorrem conforme o esperado ou apresentam alguma tendência. Para realização do teste, foi adotado um nível de significância de $p$-valor $<0.05$, ou seja, se $p$-valor $<0.05$ aceita-se $\mathbf{H}_{1}=$ As frequências observadas diferem significativamente para os diferentes grupos.

Desta forma, os dados coletados foram tabulados, interpretados, processados e analisados por meio da estatística descritiva e inferencial. Para a análise dos dados foram utilizados recursos de computação, por meio do processamento no sistema Microsoft Excel, Statistic Package for Social Sciences (SPSS) versão 24.0, todos em ambiente Windows 7.

\section{Resultados}

A Figura I indica que os principais níveis acometidos pela lesão são: T7 (6; 46\%), T8 (6; 46\%) e TI2 (6; $46 \%)$. Já a Figura 2, revela que para 9 (69\%) indivíduos a lesão é do tipo completa e a Figura 3 demonstra que maioria deles não conhece a classificação ASIA (9; 69\%). 
12 Revista Brasileira de

Sexualidade Humana

DOI: https://doi.org/10.35919/rbsh.v32il.962

Figura I - Distribuição de indivíduos com lesão medular paratletas e não paratletas, segundo o nível de lesão medular. Qual o da sua nível de lesão medular: [Níveis da lesão]

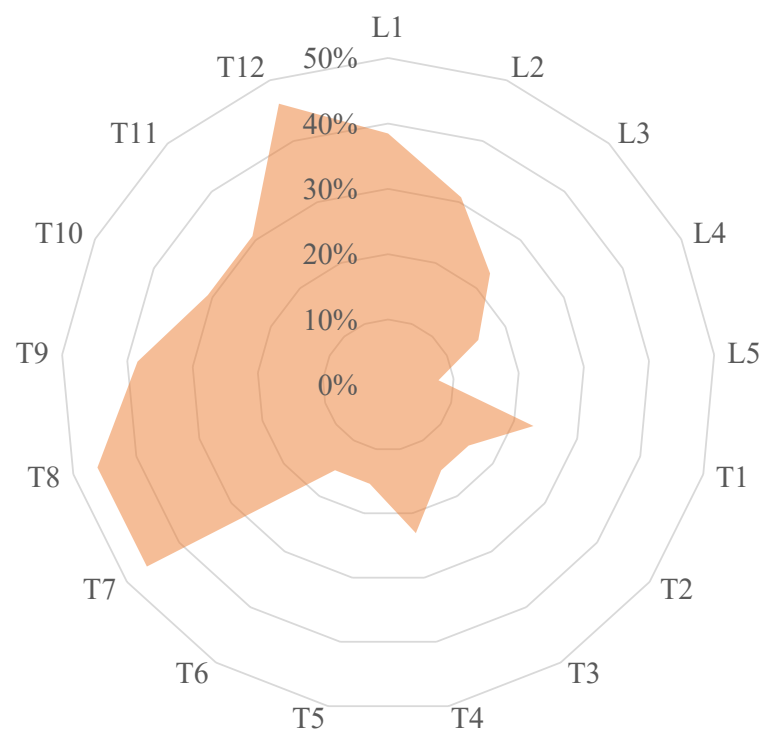

Fonte: protocolo de pesquisa (2020).

Figura 2 - Distribuição de indivíduos com lesão medular paratletas e não paratletas, segundo o tipo de lesão medular. Sua lesão é do tipo:

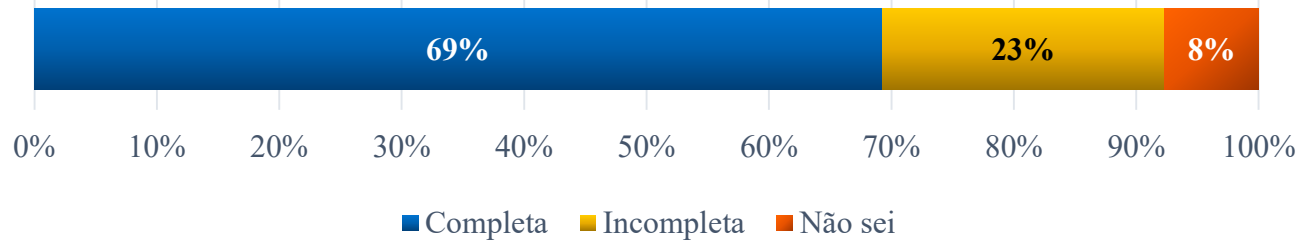

Fonte: protocolo de pesquisa (2020).

Figura 3 - Distribuição de indivíduos com lesão medular paratletas e não paratletas, segundo a classificação da ASIA.

Você Conhece a avaliação de LM da ASIA (American Spinal Injury association)? Qual a sua classificação segundo a ASIA?

\begin{tabular}{|c|c|c|c|c|c|c|c|c|c|c|}
\hline \multicolumn{4}{|c|}{$31 \%$} & \multicolumn{6}{|c|}{$69 \%$} & \\
\hline $0 \%$ & $10 \%$ & $20 \%$ & $30 \%$ & $40 \%$ & $50 \%$ & $60 \%$ & $70 \%$ & $80 \%$ & $90 \%$ & $100 \%$ \\
\hline
\end{tabular}

Fonte: protocolo de pesquisa (2020). 
A Tabela I revela que a maioria dos indivíduos, tanto aqueles que são paratletas $(6 ; 46,2 \%)$, quanto aqueles que não são paratletas $(7 ; 86 \%)$, se sentiam muito satisfeitos sexualmente antes da lesão medular. Após a lesão medular, verifica-se que somente I (14\%) indivíduo que não é paratleta se sente muito satisfeito sexualmente, 3 (50\%) indivíduos que são paratletas se sentem moderadamente satisfeitos e 3 (50\%) estão pouco satisfeitos. No grupo de praticantes de esportes, essas alterações se mostraram significativas. No grupo de praticantes de esportes, observa-se que 3 paratletas que antes da LM se sentiam muito satisfeitos, depois da $L M$ passaram a ficar pouco satisfeitos com a sua vida sexual.

Tabela I - Distribuição de indivíduos com lesão medular paratletas e não paratletas, segundo a prática de atividade física e a satisfação sexual.

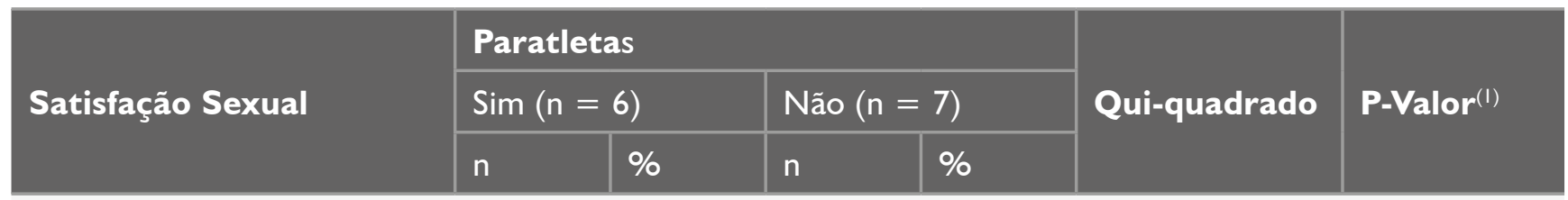

28) Quanto satisfeito(a) sexualmente você se sentia antes da LM?

\begin{tabular}{l|l|l|l|l|l|l}
\hline Muito Satisfeito(a) & 6 & $100 \%$ & 6 & $86 \%$ & \multirow{2}{*}{0.9286} & $0.3352^{\mathrm{ns}}$ \\
\cline { 1 - 6 } Pouco Satisfeito(a) & 0 & $0 \%$ & 1 & $14 \%$ & &
\end{tabular}

45) Quanto satisfeito(a) sexualmente você se sente após a LM?

\begin{tabular}{l|l|l|l|l|l|l}
\hline Moderadamente satisfeito(a) & 3 & $50 \%$ & 3 & $43 \%$ & \multirow{2}{*}{0} & \multirow{2}{*}{$0.6286^{\text {ns }}$} \\
\cline { 1 - 5 } Muito satisfeito(a) & 0 & $0 \%$ & $\mathrm{I}$ & $14 \%$ & 0.929 & \\
\hline Pouco satisfeito(a) & 3 & $50 \%$ & 3 & $43 \%$ & & \\
\hline P-Valor & $($ ()) & $\mathbf{0 . 0 0 3 *}$ & & & & \\
\hline
\end{tabular}

Fonte: protocolo de pesquisa (2020).

Nota: os resultados são baseados em linhas e colunas não vazias em cada subtabela mais interna.

(I) Teste Qui-quadrado (Wilks' $\mathrm{G}^{2}$ ) de Pearson para independência ( $\mathrm{p}$-valor $\left.<0.05\right)$.

*Valores Significativos; NS - Valores Não Significativos.

\section{Interpretação do teste:}

$\mathbf{H}_{0}$ : as frequências observadas ocorrem na mesma proporção para os diferentes grupos e categorias.

Ha: as frequências observadas diferem significativamente para os diferentes grupos e categorias.

Decisão: como o valor de $p$ computado é menor que o nível de significância alfa $=0,05$, deve-se rejeitar a hipótese nula $\mathrm{H}_{0}$ e aceitar a hipótese alternativa $\mathrm{Ha}$.

A Tabela 2 mostra que $8(61,5 \%)$ indivíduos apresentaram ajuste sexual, compreendida como a adaptação a atual condição no que tange a prática sexual, em termos físicos de nível médio com tempo médio de lesão igual a 14 anos $(\mu=14,13)$, com desvio padrão igual a $\pm 6,69$ anos. Contudo, os indivíduos com menor tempo médio de lesão $(\mu=12,00)$ apresentaram pequeno ajuste sexual em termos físicos. Estas diferenças se mostraram significativas $(p<0.05)$. Observa-se que quanto maior o tempo de lesão, maior o grau de ajuste sexual em termos físicos. 
14 Revista Brasileira de

Sexualidade Humana

DOI: https://doi.org/10.35919/rbsh.v32il.962

Tabela 2 - Tempo médio da lesão medular em paratletas e não paratletas, segundo o grau de ajuste sexual em termos físico, após a LM

\begin{tabular}{|c|c|c|c|c|c|c|}
\hline $\begin{array}{l}\text { 58). Quanto você acha que se ajustou } \\
\text { sexualmente em termos físico (capacidade } \\
\text { de realizar o que se pretende, } \\
\text { fisicamente, no sexo) após a LM? }\end{array}$ & $\mathbf{N}$ & $\%$ & $\begin{array}{l}\text { Média } \\
\text { (tempo de } \\
\text { lesão) }\end{array}$ & DesvPad & $\begin{array}{l}\text { IC de } \\
95 \%\end{array}$ & P-Valor(I) \\
\hline Grande ajuste & 3 & $23,1 \%$ & 19,67 & 8,50 & $\begin{array}{l}(10,67 \\
28,67)\end{array}$ & \multirow[t]{3}{*}{$0.046 *$} \\
\hline Médio ajuste & 8 & $61,5 \%$ & 14,13 & 6,69 & $\begin{array}{l}(8,61 ; \\
19,64)\end{array}$ & \\
\hline Pequeno ajuste & 2 & $15,4 \%$ & 12,00 & 5,66 & $\begin{array}{l}(0,98 \\
23,02)\end{array}$ & \\
\hline
\end{tabular}

DesvPad Combinado $=6,99673$

Fonte: protocolo de pesquisa (2020).

Nota: os resultados são baseados em linhas e colunas não vazias em cada subtabela mais interna.

(I) Teste t de Student ( $\mathrm{p}$-valor<0.05).

*Valores Significativos; NS - Valores Não Significativos.

Interpretação do teste:

$\mathbf{H}_{\mathbf{0}}$ : as médias observadas não são significativamente diferentes para os diferentes graus de ajuste $(p>0.05)$.

Ha: as médias observadas são significativamente diferentes para os diferentes graus de ajuste $(p<0.05)$.

Decisão: como o valor de $p$ computado é menor que o nível de significância alfa $=0,05$, deve-se rejeitar a hipótese nula $\mathrm{H}_{0}$ e aceitar a hipótese alternativa $\mathrm{Ha}$.

A Tabela 3 mostra que 7 (53,8\%) indivíduos apresentaram ajuste sexual em termos psicológicos de nível médio com tempo médio de lesão igual a 13 anos $(\mu=$ 13,00 ), com desvio padrão igual a $\pm 7,66$ anos. Somente
I indivíduo com tempo médio de lesão $(\mu=16,00)$ apresentou pequeno ajuste sexual em termos psicológicos. Essas diferenças não se mostraram significativas $(p<0.05)$.

Tabela 3 - Tempo médio da lesão medular em paratletas e não paratletas, segundo o grau de ajuste sexual em termos psicológicos, após a LM

\begin{tabular}{l|l|l|l|l|l|l}
\hline $\begin{array}{l}\text { 59). Quanto você acha que se ajustou } \\
\text { sexualmente em termos psicológicos } \\
\text { (autoestima, estima sexual, auto } \\
\text { confiança, segurança...) após a LM? }\end{array}$ & $\mathbf{N}$ & $\%$ & $\begin{array}{l}\text { Média } \\
\text { (tempo de } \\
\text { lesão) }\end{array}$ & DesvPad & $\begin{array}{l}\text { IC de } \\
\mathbf{9 5} \%\end{array}$ & P-Valor(I) \\
\hline Grande ajuste & 5 & & 17,80 & 6,30 & $\begin{array}{l}(10,68 ; \\
24,92)\end{array}$ & $0.535 \mathrm{~ns}$ \\
\hline Médio ajuste & 7 & $38,5 \%$ & & 13,00 & 7,66 & $\begin{array}{l}(6,98 ; \\
19,02)\end{array}$ \\
\hline Pequeno ajuste & 1 & $53,8 \%$ & & 16,00 & $*$ & $\begin{array}{l}(0,08 ; \\
31,92)\end{array}$ \\
\hline
\end{tabular}

DesvPad Combinado $=7,14703$

Fonte: protocolo de pesquisa (2020).

Nota: os resultados são baseados em linhas e colunas não vazias em cada subtabela mais interna.

(I) Teste t de Student ( $\mathrm{p}$-valor<0.05).

*Valores Significativos; NS - Valores Não Significativos.

Interpretação do teste:

$\mathbf{H}_{\mathbf{0}}$ : as médias observadas não são significativamente diferentes para os diferentes graus de ajuste $(p>0.05)$.

Ha: as médias observadas são significativamente diferentes para os diferentes graus de ajuste $(p<0.05)$.

Decisão: como o valor de $p$ computado é menor que o nível de significância alfa $=0,05$, deve-se rejeitar a hipótese nula $\mathrm{H}_{0}$ e aceitar a hipótese alternativa $\mathrm{Ha}$. 
A Tabela 4 indica que 5 (38\%) indivíduos receberam de forma moderada algum tipo de orientação ou aconselhamento sexual após a lesão medular e 5 (38\%) indivíduos não receberam nenhuma orientação ou aconselhamento sexual após a lesão medular; 9 (69\%) indivíduos afirmam que aceitariam receber orientação ou aconselhamento sexual; 6 (46\%) indivíduos afirmam que seu parceiro(a) não recebeu algum tipo de aconselhamento sexual; 4 (3l\%) indivíduos declararam que o recebimento de algum tipo de orientação melhoraria muito a qualidade da sua vida sexual e para 4 (3l\%) indivíduos melhoraria moderadamente.

Tabela 4 - Distribuição de indivíduos com lesão medular paratletas e não paratletas, segundo o tipo de atividades praticadas na vida sexual ativa

\section{Vida Sexual Ativa pós lesão}

n

$\%$

Qui-quadrado P-Valor(1)

54) Você recebeu algum tipo de orientação ou aconselhamento sexual após a LM?

\begin{tabular}{l|l|l|l|l}
\hline Moderado & 5 & $38 \%$ & 3,923 & 0,270 \\
\hline Muito & 2 & $15 \%$ & & \\
\hline Nada & 5 & $38 \%$ & & \\
\hline Pouco & 1 & $8 \%$ & & \\
\hline
\end{tabular}

55) Se NÃO, você aceitaria receber orientação ou aconselhamento sexual após a LM (se não tivesse custo financeiro)?

\begin{tabular}{l|l|l|l|l}
\hline $\operatorname{Sim}$ & 9 & $69 \%$ & 1,923 & 0,166 \\
\hline Não & 4 & $31 \%$ & & \\
\hline
\end{tabular}

56) E seu(sua) parceiro(a), recebeu algum tipo de aconselhamento sexual?

\begin{tabular}{l|l|l|l|l}
\hline Nada & 6 & $46 \%$ & 1,077 & 0,584 \\
\hline Não tenho parceiro(a) ou Não sei. & 3 & $23 \%$ & & \\
\hline Pouco & 4 & $31 \%$ & & \\
\hline
\end{tabular}

57) Você acha que algum tipo de orientação melhoraria a qualidade da sua vida sexual?

\begin{tabular}{l|l|l|l|l}
\hline Melhoraria Moderadamente & 4 & $31 \%$ & 0,846 & 0,838 \\
\hline Melhoraria Muito & 4 & $31 \%$ & & \\
\hline Melhoraria Pouco & 3 & $23 \%$ & & \\
\hline Não Melhoraria & 2 & $15 \%$ & & \\
\hline
\end{tabular}

Fonte: protocolo de pesquisa (2020).

Nota: os resultados são baseados em linhas e colunas não vazias em cada subtabela mais interna.

(I) Teste Qui-quadrado (Wilks' $\mathrm{G}^{2}$ ) de Pearson para independência ( $\mathrm{p}$-valor $<0.05$ ).

*Valores Significativos; NS - Valores Não Significativos.

Interpretação do teste:

$\mathbf{H}_{0}$ : as frequências observadas ocorrem na mesma proporção para os diferentes grupos e categorias.

Ha: as frequências observadas diferem significativamente para os diferentes grupos e categorias.

Decisão: como o valor de $p$ computado é menor que o nível de significância alfa $=0,05$, deve-se rejeitar a hipótese nula $\mathrm{H}_{0}$ e aceitar a hipótese alternativa $\mathrm{Ha}$.

\section{Discussão}

O impacto negativo na qualidade de vida sexual da pessoa que sofreu LM foi relatado na literatura. Segundo o estudo do Pascual (2019), a lesão da medula espinhal pode causar sérios danos à saúde sexual e ao comportamento sexual, resultando em diminuição da autoestima, isolamento social e risco de depressão, reduzindo assim a qualidade de vida. A disfunção sexual é uma evidência comum após lesão da medula espinhal, como orgasmo retardado, distúrbios eréteis ou de ejaculação, anormalidades de sêmen e infertilidade

Essas limitações, segundo Sunilkumar, Boston e Rajagopal (20I5), são devido à falta de conscientização pública, falta de necessidades sexuais dos lesados medulares e consciência insuficiente da vida sexual dos paraplégicos 
como um aspecto importante de sua saúde geral, ou seja, a falta de informação por parte dos indivíduos com lesão faz com que não busquem o tratamento para melhora da função sexual. Ainda mais que, segundo Araújo e Silva (2018), os portadores da LM que vão buscar tratamento, priorizam a função motora sobretudo a função sexual.

Este estudo revela que a inclusão social de atletas é de grande significância, pois a qualidade de vida melhora devido à interação social, já que reduz a depressão, a ansiedade e $\circ$ estresse, a elevação da autoestima, o aumento da segurança do comportamento sexual e, consequentemente, dispõe de uma melhoria da qualidade de vida sexual. No estudo, $50 \%$ dos atletas permaneceram, com a vida sexual, moderadamente satisfeito após a LM.

Foram identificados estudos que relacionam o grau da lesão com a qualidade de vida sexual dos indivíduos após $L M$, contribuindo com a presente pesquisa que identificou que, dependendo do grau da lesão de cada indivíduo, a qualidade de vida sexual pode ser ainda mais afetada. $O$ estudo Kazum e outros (20I8), relata que os indivíduos que sofreram lesão transversal completa na medula espinhal acima de L2 não possuem ereção psicogênica, prevalece apenas a ereção reflexa, pois as vias simpáticas psicogênicas são mais frequentemente prejudicadas do que as vias reflexogênicas parassimpáticas; quando a lesão é entre os segmentos L2-SI, a ereção reflexa e psicogênica são preservadas, e quando a lesão ocorre no cone medular, não tem a reflexa, porém tem a psicogênica. Na lesão longitudinal não ocorre a psicogênica nem reflexa, oposto das lesões incompletas, em que a ereção é frequentemente possível.

Park e outros (2016), realizaram uma pesquisa com 50 participantes do sexo masculino e feminino, na qual discutiram o impacto da bexiga urinária, intestino e disfunção sexual no estado de saúde de pessoas com Lesão Medular. O estudo destacou que os indivíduos com lesão medular completa (ASIA A) são mais propícios a ter disfunção sexual, ou até mesmo disfunções múltiplas. Com isso concluiu-se que o Grau A da lesão estabelecida pela Associação Americana de Lesões Medulares (ASIA) provoca maiores malefícios à qualidade de vida sexual. Dessa forma, corroborando a ideia do presente estudo que identificou que em $69 \%$ dos indivíduos participantes a lesão é do tipo completa, em função disso, grande parte deles possui uma certa debilidade na prática sexual.

Os estudos anteriores reforçam os resultados obtidos nesta pesquisa, apontando que os principais níveis acometidos pela lesão são: T7 (6; 46\%), T8 (6; 46\%) e TI2 (6; 46\%), sendo que em 9 (69\%) indivíduos a lesão é do tipo completa e a maioria não conhece a classificação $\operatorname{ASIA}(9 ; 69 \%)$, que atualmente a maioria $(8 ; 62 \%)$ dos participantes da pesquisa têm vida sexual ativa, entende-se que a qualidade de vida sexual está inteiramente associada ao grau em que o indivíduo foi acometido, lesões completas (ASIA A) apresentam maiores perdas na função sexual e ressalta a falta de conhecimento sobre a American Spinal Injury Association (ASIA).

Galati e outros (20I4) avaliaram em sua pesquisa a sexualidade relacionada à qualidade de vida de homens. Participaram do estudo dez indivíduos, com média de idade de 52,8 anos. Foram observados a partir da escala The Golombok-Rust Inventory of Sexual Satisfaction (GRISS) os fatores para avaliar a Insatisfação Sexual Masculina (ISM). Cinco pacientes atingiram escore superior a cinco pontos, identificando a presença de problemas sexuais.

No presente estudo, os resultados mostraram que os sujeitos investigados na pesquisa que não praticam esporte, a maioria (86\%), não possuem ou possuem pequeno grau de satisfação sexual e, dos que praticam a atividade física, $50 \%$ da amostra, sentem-se muito satisfeito e $50 \%$ pouco satisfeitos. Essas mudanças se mostraram significativas. No grupo de praticantes de esportes, observou-se que 3 atletas estavam muito satisfeitos antes da LM, e diminuíram a satisfação com sua vida sexual após $L M$.

Os dados indicaram que $8(61,5 / \%)$ dos 13 indivíduos participantes da pesquisa possuem um tempo médio de lesão igual a 14 anos $(\mu=14,13)$ e declaram um ajuste médio sexual em termos físicos (capacidade de realizar o que se pretende, fisicamente, no sexo). Entretanto, os indivíduos com menor tempo médio de lesão $(\mu=12,00)$ apresentaram pequeno ajuste sexual em termos físicos. Estes resultados revelam que o tempo da lesão é diretamente proporcional ao ajuste sexual em termos físicos.

O estudo de Cardoso e outros (2018) descreve que o tempo da lesão está associado à qualidade de vida sexual, pois constatou que a satisfação sexual está ligada ao tempo de reabilitação deste individuo após a lesão. Quanto maior o tempo de lesão medular, maior será o período de reajuste sexual, percebendo que a satisfação sexual apresenta-se reduzida no recém lesionado, e com a duração da reabilitação tende aumentar, mesmo que este indivíduo não se reedifique completamente.

Já em termos psicológicos (autoestima, estima sexual, autoconfiança, segurança...), 7 (53,8\%) indivíduos apresentaram ajuste sexual médio com tempo de lesão igual a I 3 anos. Contudo, apenas I indivíduo com tempo médio de lesão $(\mu=16,00)$ apresentou pequeno ajuste sexual em termos psicológicos. Tais discrepâncias não se expressam significativas $(p<0.05)$.

Diante disso, a presente pesquisa corrobora com o estudo efetuado por Galati e outros (20।4), o qual inferiu que, dentre as dimensões avaliadas, o domínio da saúde mental obteve a menor média, ressaltando que o componente físico possui uma maior queixa por parte dos indivíduos.

A análise dos dados obtidos, com relação à existência de aconselhamento sexual pelos profissionais da saúde às pessoas com lesão medular e seus parceiros, indicou que dos 13 indivíduos amostrados, 5 (38\%) não receberam 
nenhum tipo de orientação ou aconselhamento sexual, 5 (38\%) receberam aconselhamento sexual de forma moderada e 6 (46\%) indivíduos afirmam que seu parceiro(a) não recebeu algum tipo de aconselhamento sexual.

Outro estudo, em Goiânia-GO, concluiu que pessoas com LM necessitam de instruções quanto às mudanças na sexualidade pós-Lesão Medular, no que se refere a readaptação de posicionamento, excitação, desejo, utilização de dispositivos que auxiliem no ato sexual, e que a falta de conhecimento pode provocar ansiedade, medo e frustração, trazendo uma visão de não ser capaz de realizar a prática sexual devidos às limitações (MAGALHÃES; SOUZA; OLIVEIRA, 2017).

$\mathrm{Na}$ Holanda, Pascual e outros (2019), realizaram uma pesquisa com um grupo de 25 enfermeiros, a qual evidenciou que apenas dois entrevistados abordaram ativamente a sexualidade com seus pacientes designados durante a reabilitação. Vinte e um participantes explicaram que só abordaram ativamente $o$ assunto com pacientes selecionados ou falavam sobre o assunto caso o indivíduo com LM iniciasse a conversa. Duas enfermeiras não discutiram 0 assunto. Diante disso, as barreiras impostas pelo tabu que é a sexualidade é um dos aspectos que tem prejudicado significativamente a qualidade de vida sexual dessas pessoas com LM, devido a muitos profissionais ainda hoje se sentirem desconfortáveis para abordar esse assunto com seus pacientes.

Embora a vasta gama de informações detalhadas obtidas por meio do questionário, algumas limitações foram encontradas na presente pesquisa, como a baixa quantidade amostral, pois, devido a pandemia do COVID19 , a coleta de dados presencial se tornou dificultosa, visto que muitos dos indivíduos não retornaram as atividades em razão dos riscos. Ademais, o baixo índice de adesão do questionário on-line foi outro fator limitador para uma melhor percentagem amostral. Além disso, a escassez de pesquisas abordando a sexualidade de paratletas tornou-se uma deficiência do atual estudo, posto que limitou a comparação de informações quanto a esse assunto.

\section{Conclusão}

O estudo demonstrou que houve diferença significativa antes da LM para o depois da lesão na diminuição da qualidade de vida sexual dos dois grupos. Vale ressaltar que no grupo dos não paratletas a porcentagem foi menor do que no grupo dos paratletas, no entanto, não houve significância estatística. Dessa forma, a importância de aconselhamento para esses indivíduos torna-se indispensável para melhora da qualidade de vida sexual. Além disso, indica-se a necessidade de estudos mais específicos na área da saúde sexual com enfoque em lesão medular e o desporto, que visem pesquisas mais aprofundadas, com maior número amostral de sujeitos investigados.

\section{Referências}

ARAÚjO E SILVA, R. et al. Atividade sexual na lesão medular: construção e validação de cartilha educativa. Acta Paul de Enfermagem, v. 3I, n. 3, p. 255-264, jun. 2018. Disponível em: https://doi. org/I0.I590/I982-0I9420I800037. Acesso em: II fev. 202।.

CARDOSO, F. L. et al. Fatores associados à satisfação sexual de homens com lesão medular. Fisioterapia e Pesquisa, São Paulo, v. 25, n. I, jan./mar. 2018. Disponível em: https://doi. org/I0.1590/I809-2950/I67426250I20I8 . Acesso em: II fev. 202I.

FIDLER, A.; SCHMIDT, M.; VAUHNIK, J. Ways that people with a chronic spinal cord injury participate in sport in the Republic of Slovenia. Journal of Physical Education and Sport, Polônia, v. 17, n. 3, p. 1892-1898, Sept. 2017. Disponível em: https://www. researchgate.net/deref/http\%3A\%2F\%2Fdx. doi.org\%2FI0.7752\%2Fjpes.20I7.03। 84 . Acesso em: II fev. 202I.

GALATI, M. C. R et al. Sexualidade e qualidade de vida em homens com dificuldades sexuais. Psico-USF, Bragança Paulista, v. 19, n. 2, p. 243-252, maio/ago. 20I4. Disponível em: https://www.scielo.br/scielo.php?pid=SI4 I 3$827|20| 4000200007$ \&script $=$ sci abstract\&tlng =pt . Acesso em: II fev. 202I .

JAMES, S. L. et al. Global, regional, and national burden of traumatic brain injury and spinal cord injury, 1990-2016: a systematic analysis for the Global Burden of Disease Study 2016. The Lancet Neurology, Seattle, v. I8, n. I, p 56-87, Jan. 2019. Disponível em: https://www.sciencedirect. com/science/article/pii/S I 4744422 I8304I 50 . Acesso em: II fev. 202I.

KRASSIOUKOV, A.; ELLIOTT, S. Neural Control and Physiology of Sexual Function: Effect of Spinal Cord Injury. Topics in spinal cord injury Rehabilitation. Vancouver, v. 23, n. I, p. I-I0. 2017. Disponível em: https://pubmed.ncbi.nlm.nih. gov/29339872/. Acesso em: II fev. 202I.

MAGALHÃES, M. A.; SOUZA, J. C.; OLIVEIRA, F. M. Orientação sexual para pessoa com lesão medular. Revista Brasileira de Sexualidade Humana, v. 28, n. I, p. 23-30, 20I7. Disponível em: https://doi.org/l0.35919/rbsh.v28il.5 . Acesso em: II fev. 202I. 
18 Revista Brasileira de

Sexualidade Humana

DOI: https://doi.org/I0.35919/rbsh.v32il.962

MIRANDA, E. et al. Evaluation of Sexual Dysfunction in men with Spinal Cord Injury using the Male Sexual Quotient. Archives of physical medicine and rehabilitation, São Paulo, v. 97, n. 6, p. 947-952, Jan. 2016. Disponível em: https:// pubmed.ncbi.nlm.nih.gov/26827830/ . Acesso em: II fev. 202I.

PARK, S. E. et al. Impact of bladder, bowel and sexual dysfunction on health status of people with thoracolumbar spinal cord injuries living in the community. The Journal of Spinal Cord Medicine, Estados Unidos, v. 40, n. 5, p. 548-559, Aug. 2016. Disponível em: https://www.ncbi.nlm. nih.gov/pmc/articles/PMC58I5I54/. Acesso em: II fev. 202 I.

PASCUAL, A. et al. Sexuality as part of rehabilitation? A qualitative study on the perceptions of rehabilitation nurses on discussing patient sexuality during clinical rehabilitation. Disability and Rehabilitation. Inglaterra, v. I5, p. I-8, Oct. 2019. Disponível em: https:// pubmed.ncbi.nlm.nih.gov/31613157/ . Acesso em: Il fev. 202 I.

SUNILKUMAR, M. M.; BOSTON, P.; RAJAGOPAL, $M$. R. Sexual Functioning in Men Living with a Spinal Cord Injury-A Narrative Literature Review. Indian Journal Palliat Care. India, v. 21, n. 3, p. 274-28I, Sept./Dec. 20I5. Disponível em: https://www.ncbi.nlm.nih.gov/pmc/articles/ PMC4617033/ . Acesso em: II fev. 202I.

WALTER, M.; KRASSIOUKOV, A. Autonomic Nervous System in Paralympic Athletes with Spinal Cord Injury. Physical Medicine and Rehabilitation Clinics of North America. Tennessee, v. 29, n. 2, p. 245-266, May. 20I9. Disponível em: https://pubmed.ncbi.nlm.nih.gov/29627087/ . Acesso em: II fev. 202I. 